\title{
The NStED Stellar and Exoplanet Hosting Star Service
}

\author{
S. Ramirez ${ }^{2,4,5}$, B. Ali ${ }^{2,4}$, R. Baker $^{2,4}$, G. B. Berriman ${ }^{1,2,4}$, K. von \\ Braun $^{1,4}$, N-M. Chiu ${ }^{2,4}$, D. R. Ciardi ${ }^{1,4}$, J. Good ${ }^{2,4}$, S. R. Kane ${ }^{1,4}$, \\ A. C. Laity ${ }^{2,4}$, D. L. McElroy ${ }^{2,4}$, S. Monkewitz ${ }^{2,4}$, A. N. Payne ${ }^{1,2,4}$, \\ M. Schmitz ${ }^{2,4}$, J. R. Stauffer ${ }^{3,4}$, P. L. Wyatt ${ }^{1,2,4}$, and A. Zhang ${ }^{2,4}$ \\ ${ }^{1}$ Michelson Science Center; ${ }^{2}$ Infrared Processing and Analysis Center; ${ }^{3}$ Spitzer Science \\ Center; ${ }^{4}$ California Institute of Technology; ${ }^{5}$ email: solange@ipac.caltech.edu
}

\begin{abstract}
The NASA Star and Exoplanet Database (NStED) is a general purpose stellar archive with the aim of providing support for NASA's planet finding and characterization goals, stellar astrophysics, and the planning of NASA and other space missions. There are two principal components of NStED: a database of (currently) 140,000 nearby stars and exoplanet-hosting stars, and an archive dedicated to high precision photometric surveys for transiting exoplanets. We present a summary of the NStED stellar database, functionality, tools, and user interface. NStED currently serves the following kinds of data for 140,000 stars (where available): coordinates, multiplicity, proper motion, parallax, spectral type, multiband photometry, radial velocity, metallicity, chromospheric and coronal activity index, and rotation velocity/period. Furthermore, the following derived quantities are given wherever possible: distance, effective temperature, mass, radius, luminosity, space motions, and physical/angular dimensions of habitable zone. Queries to NStED can be made using constraints on any combination of the above parameters. In addition, NStED provides tools to derive specific inferred quantities for the stars in the database, cross-referenced with available extra-solar planetary data for those host stars. NStED can be accessed at http://nsted.ipac.caltech.edu.
\end{abstract}

\section{The NStED Services}

The NASA Star and Exoplanet Database (NStED) is dedicated to collecting and serving vital published data involved in the search for and study of extrasolar planets and their host stars. The stellar and exoplanet services provide access to stellar parameters of potential exoplanet bearing stars along with exoplanet parameters. The stellar services provided by NStED include the following:

- Data related to relatively bright nearby stars.

- The ability to query for individual stars or search by stellar/planetary parameters.

- Published images, spectra, and time series data related to the stars in the database. Complementary to this are the exoplanet services, which includes the following:

- General data and pubished parameters for the known exoplanets and host stars.

- Photometric and radial velocity data related to the known exoplanets.

- A dedicated interface related to exoplanet transit surveys (see companion paper by von Braun et al. (2008) in these proceedings).

Figure 1 shows an example plot produced using the data served by NStED.

\section{Stellar Content for NStED}

NStED's stellar and exoplanet content is composed of published tabular data, derived and calculated quantities, and associated data including images, spectra, and time series. 


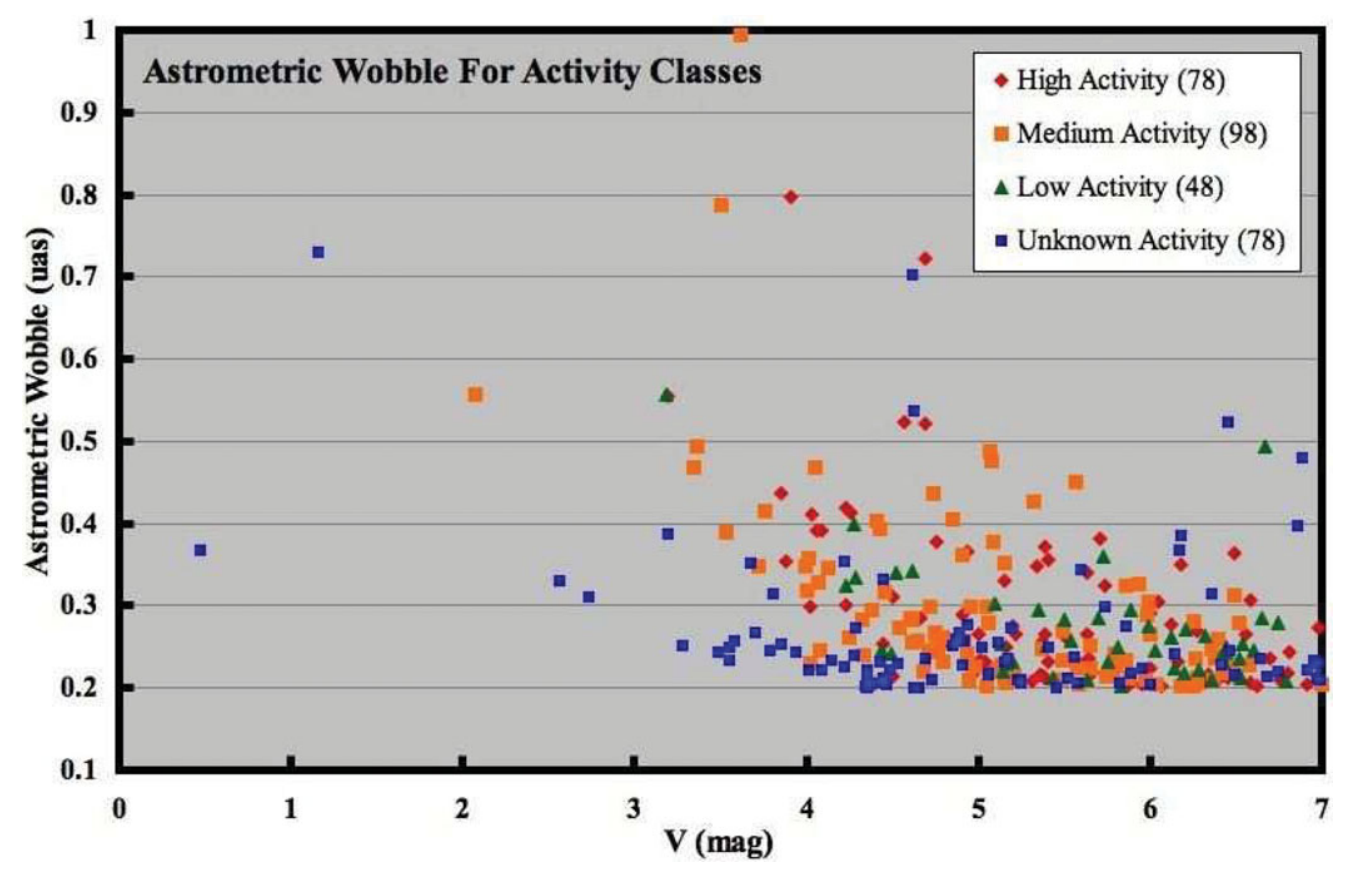

Figure 1. Plot of predicted astrometric wobble for an Earth-sized planet in the habitable zone vs. the apparent V magnitude of the stars, generated using data served by NStED. The stars are sorted by activity level estimates from the R'(HK) index, S index, and X-ray luminosity.

Table 1. Summary of stellar content within NStED.

\begin{tabular}{lll}
\hline Published Parameters & Derived Parameters & Associated Data \\
\hline Position, Distances & Temperature & Images \\
Kinematics & Luminosity & Spectra \\
Photometry, Colors & Radius & \\
Spectral Type & Mass & \\
Luminosity Class & LSR Space Motion & \\
Metallicity & \\
Rotation & \\
Activity Indicators & \\
Variability & \\
Multiplicity & \\
\hline
\end{tabular}

An example spectrum from the N2K consortium (Fischer et al. 2005) contained within NStED is shown in Figure 2. NStED's core set of stars is derived from the Hipparcos, Gliese-Jahreiss, and Washington Double Star catalogs. The total number of Hipparcos and Gliese-Jahreiss stars within NStED is approximately 140,000. A summary of the stellar parameters and data within NStED is shown in Table 1. NStED currently supports complex multi-faceted queries on approximately 75 astrophysical stellar and exoplanet parameters.

\section{Exoplanet Content for NStED}

In order to facilitate future exoplanet studies, NStED maintains an up-to-date list of exoplanetary systems and associated stellar data by monitoring daily the literature and 


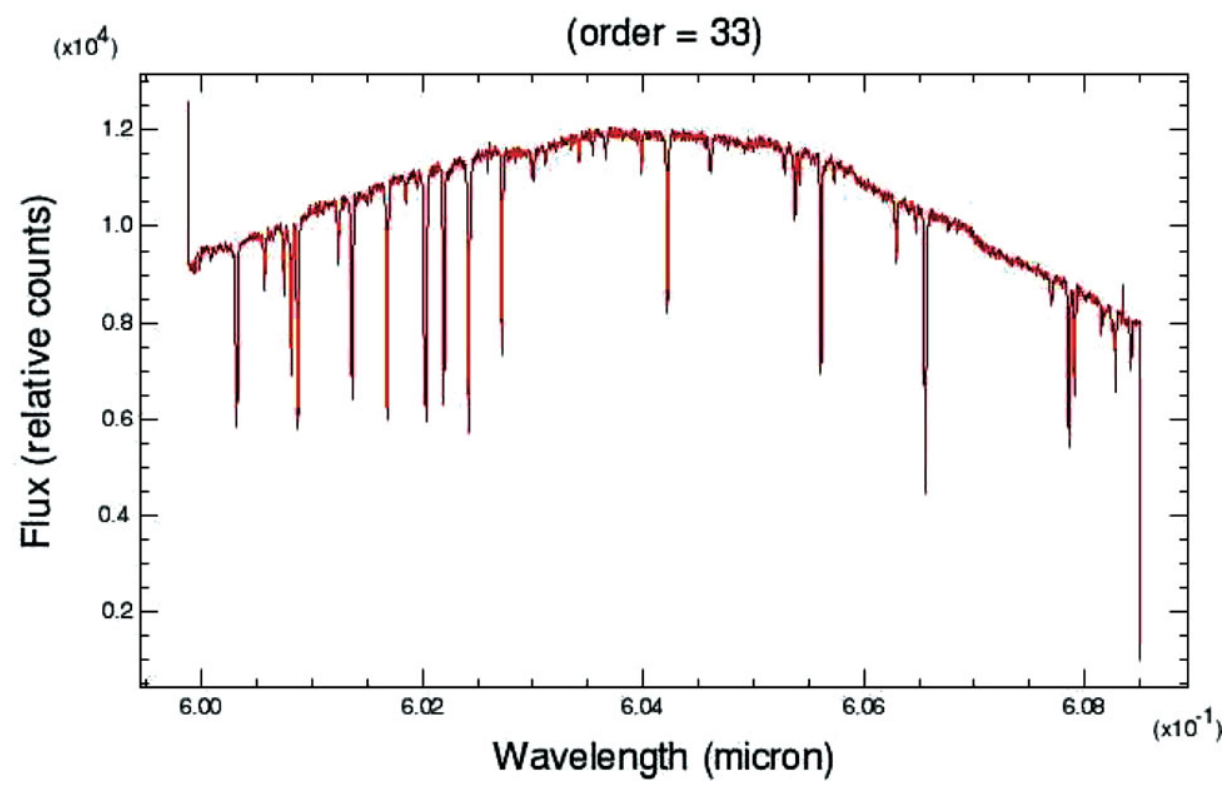

Figure 2. N2K spectrum of HD 804.

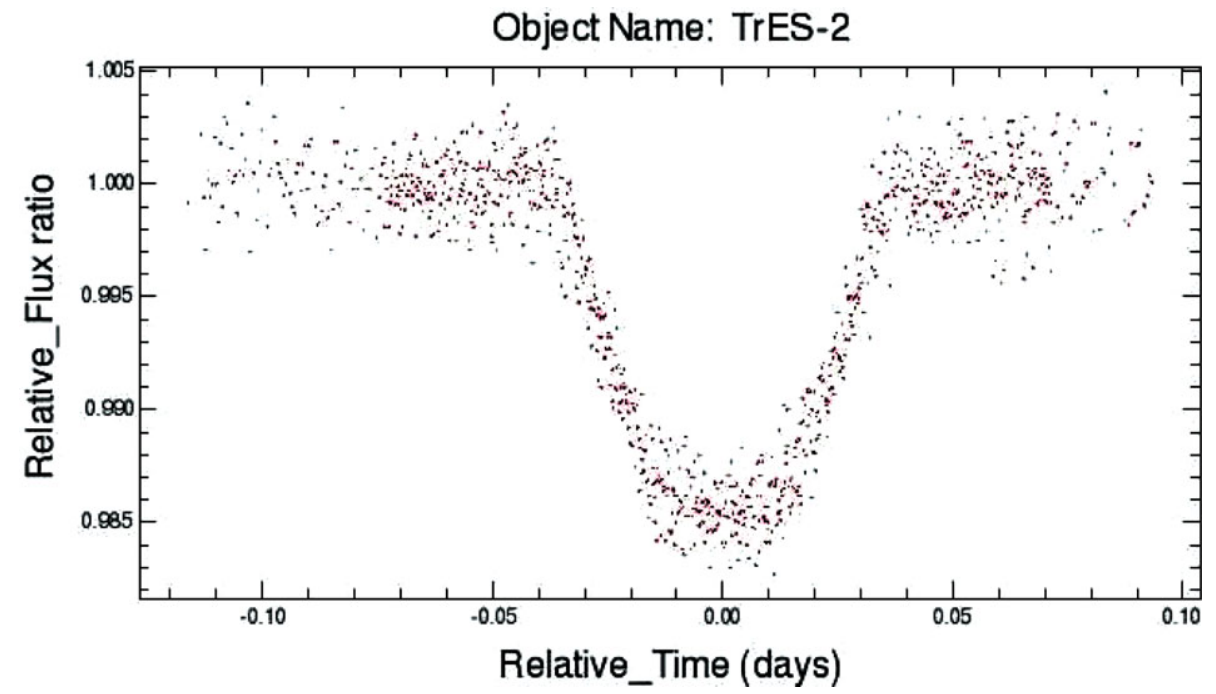

Figure 3. Lightcurve of the transiting exoplanet TrES-2 (O'Donovan et al. 2006).

making weekly updates to the database. These data include high-precision lightcurves, such as the lightcurve for the transiting planet TrES-2 shown in Figure 3. The predicted signatures of exoplanets are also calculated to aid users in selection of stars appropriate for planet searching and characterization. The exoplanet signature predictions include habitable zone sizes, astrometric and radial velocity wobbles, and transit depths. A summary of the exoplanet parameters and data within NStED is shown in Table 2. 
Table 2. Summary of exoplanet content within NStED.

\begin{tabular}{lll}
\hline Published Parameters & Predicted Parameters & Associated Data \\
\hline Number of Planets & Habitable Zone & High Contrast Images \\
Planetary Mass & Astrometric Wobble & Lightcurves \\
Orbital Period & Radial Velocity Wobble & \\
Orbital semi-major axis & Earth V Magnitude & \\
Orbital Eccentricity & Earth 10 $\mu$ m flux density & \\
Link to entry in the & & \\
Exoplanet Encyclopaedia & & \\
\hline
\end{tabular}

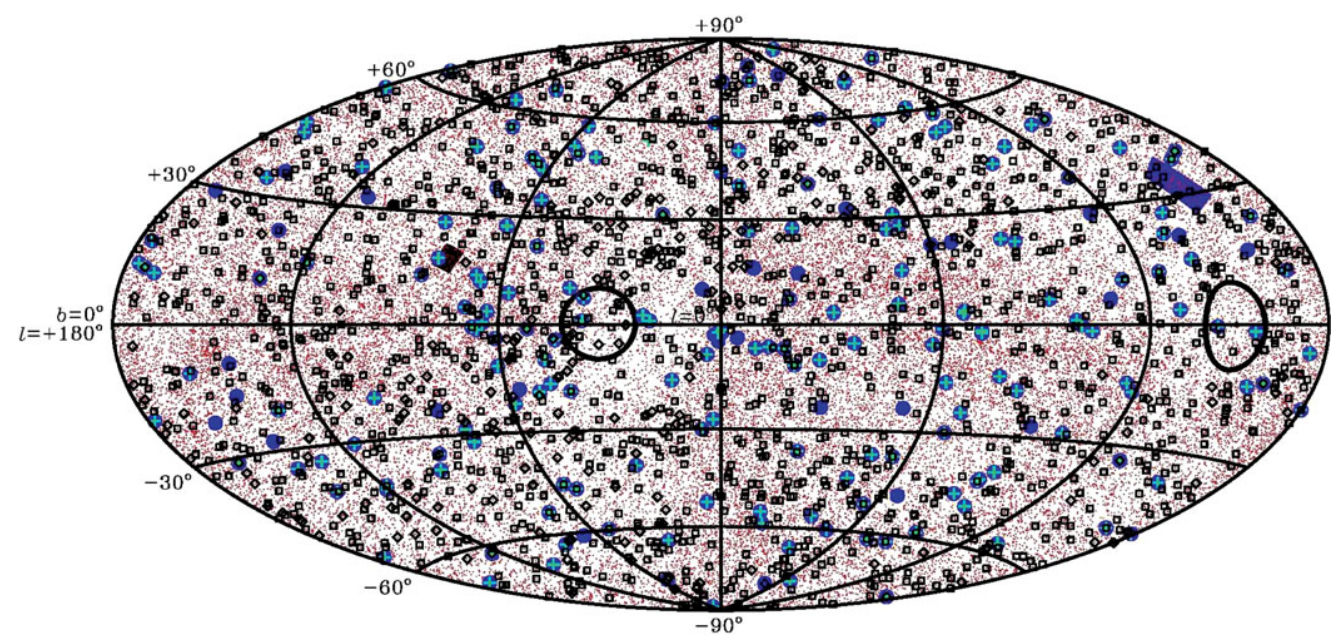

Figure 4. Aitoff projection of the contents of NStED. Small dots: dwarf stars (for clarity, the giant stars are not plotted); large dots: exoplanet hosting stars; large plus signs: stars with radial velocity curves or photometric lightcurves; open squares/diamonds: stars with images/spectra. For an explanation of the remaining features, see companion paper on the NStED Exoplanet Transit Survey Service by von Braun et al. in this volume.

\section{Summary}

The NStED Stellar and Exoplanet Hosting Star Service provides access to data relevant to exoplanet host stars and bright stars from major catalogues. The search query tools and cross-referencing capabilities make this a powerful engine through which to aid in exoplanet survey programs. NStED provides not only tabular data, but also a wide variety of associated data including images, spectra, and time series (radial velocity and photometric) obervations. NStED is continually updated to reflect the latest results in the literature and to provide published data access to the broader astronomical community. NStED is accessible via http://nsted.ipac.caltech.edu.

\section{References}

Fischer, D. A., et al. 2005, ApJ, 620, 481

O’Donovan, F. T., et al. 2006, ApJ, 651, L61 